\title{
BMJ Global Health The 117 call alert system in Sierra Leone: from rapid Ebola notification to routine death reporting
}

Charles Alpren, ${ }^{1}$ Mohamed F Jalloh, ${ }^{2}$ Reinhard Kaiser, ${ }^{1}$ Mariam Diop, ${ }^{3}$ SAS Kargbo, ${ }^{4}$ Evelyn Castle, ${ }^{3}$ Foday Dafae, ${ }^{4}$ Sara Hersey, ${ }^{1}$ John T Redd, ${ }^{2}$ Amara Jambai $^{4}$

To cite: Alpren C, Jalloh MF Kaiser $\mathrm{R}$, et al. The 117 call alert system in Sierra Leone: from rapid Ebola notification to routine death reporting. BMJ Glob Health 2017;2:e000392. doi:10.1136/ bmjgh-2017-000392

Received 3 May 2017 Revised 12 July 2017 Accepted 14 July 2017

\section{CrossMark}

${ }^{1}$ Centers for Disease Control and Prevention, Freetown, Sierra Leone

${ }^{2}$ Division of Global Health Protection, Center for Global Health, Centers for Disease Control and Prevention, Atlanta, Georgia, USA

${ }^{3}$ eHealth Africa, Freetown, Sierra Leone

${ }^{4}$ Ministry of Health and Sanitation, Freetown, Sierra Leone

Correspondence to Dr Charles Alpren; nra8@cdc.gov

\section{ABSTRACT}

A toll-free, nationwide phone alert system was established for rapid notification and response during the 2014-2015 Ebola epidemic in Sierra Leone. The system remained in place after the end of the epidemic under a policy of mandatory reporting and Ebola testing for all deaths, and, from June 2016, testing only in case of suspected Ebola. We describe the design, implementation and changes in the system; analyse calling trends during and after the Ebola epidemic; and discuss strengths and limitations of the system and its potential role in efforts to improve death reporting in Sierra Leone. Numbers of calls to report deaths of any cause (death alerts) and persons suspected of having Ebola (live alerts) were analysed by province and district and compared with numbers of Ebola cases reported by the WHO. Nearly 350000 complete, non-prank calls were made to 117 between September 2014 and December 2016. The maximum number of daily death and live alerts was 9344 (October 2014) and 3031 (December 2014), respectively. Call volumes decreased as Ebola incidence declined and continued to decrease in the post-Ebola period. A national social mobilisation strategy was especially targeted to influential religious leaders, traditional healers and women's groups. The existing infrastructure and experience with the system offer an opportunity to consider long-term use as a death reporting tool for civil registration and mortality surveillance, including rapid detection and control of public health threats. A routine social mobilisation component should be considered to increase usage.

\section{INTRODUCTION}

The first case of Ebola virus disease (Ebola) in Sierra Leone was detected in May 2014. ${ }^{1}$ The associated epidemic, which heavily affected Guinea, Liberia and Sierra Leone, resulted in over 28000 cases and 11000 deaths $^{1}$ and was exacerbated by pre-existing deficiencies in public health infrastructure. ${ }^{2}$ Factors that contributed to spread of Ebola included delays in seeking medical care coupled with traditional burial rituals that involved physical contact with corpses. ${ }^{34}$ In addition, the prolonged epidemic may have had other

\section{Key questions}

What is already known about this topic?

- Prompt identification of suspected Ebola cases and deaths contributed to the containment of the 2014-2015 epidemic in West Africa.

- In Sierra Leone, a toll-free telephone system was used for reporting sick people and deaths occurring in communities during the Ebola response as part of containment efforts.

\section{What are the new findings?}

- We provide a comprehensive description of the 117 call system, including an explanation of how it was used before, during and after the Ebola response in Sierra Leone.

- For the first time, we summarise trends in call volumes against Sierra Leone's Ebola epidemic curve and timeline of major social mobilisation activities

-We discuss the system's design, implementation, infrastructure, personnel and costs.

Recommendations for policy

- Understanding how the 117 call system was used during and after the Ebola response can inform potential uses during non-outbreak conditions as well as for future outbreak responses.

- Practical lessons learnt from the implementation of the system in Sierra Leone may be applicable to other similar settings in the subregion.

adverse effects on the use of essential primary care services. ${ }^{5}$

A national alert system with a single, national toll-free phone number, 117, was introduced in Sierra Leone in August $2014^{6}$ to facilitate prompt identification, investigation, isolation and testing of potential Ebola cases and deaths. ${ }^{78}$ During this period, the government maintained a policy of mandatory reporting and Ebola testing for all deaths. The 117 system remained an integral response component during the enhanced surveillance period from 
the official end of the Ebola epidemic in Sierra Leone in November 2015 until June 2016. The 117 system continues to be used to help detect possible Ebola flare-ups, including those that may be linked to viral persistence in male Ebola survivors. ${ }^{9}$

In this paper, we describe the 117 system, analyse numbers of death-alert and live-alert calls by region from 1 September 2014 to 31 December 2016, and compare call trends with daily numbers of confirmed Ebola cases from published WHO situation reports. We discuss strengths and limitations of the system and its potential long-term role in efforts to improve death reporting and enhance International Health Regulations implementation in Sierra Leone, and thereby enhance global health security.

Our analysis of calls was restricted to 'complete calls' made for death alerts $(\mathrm{n}=248789)$ and live alerts $(\mathrm{n}=95136)$. We excluded calls that were 'prank' (defined as any call that was clearly nefarious) or 'incomplete' (defined as any call that dropped out before adequate information could be conveyed). Overall, calls that were prank or incomplete made up $>50 \%$ of all calls placed to 117. All calls deemed prank or incomplete received a follow-up call from the call centre to ensure no legitimate calls were lost. We did not further assess the role of calls that were prank or incomplete, and network limitations on the overall use of the system. Furthermore, as the purpose of this analysis was to examine trends in overall call volumes, we did not attempt to eliminate duplicate calls. Assessing caller profiles or behavioural patterns during various stages during and after the epidemic was beyond the scope of this report.

\section{SYSTEM DESIGN AND IMPLEMENTATION}

In August 2014, the Government of Sierra Leone repurposed an existing national toll-free hotline for reporting of potential Ebola cases and deaths in communities. The telephone number ' 117 ' could be used at no charge to the caller from any major mobile telecommunication network in the country. Calls were made to alert authorities of sick persons requiring isolation and Ebola testing (live alerts), and deaths from any cause (death alerts), which under national policy required safe and dignified burials performed by trained teams. ${ }^{10}$ Calls made to 117 were answered at a national centre located in the capital, Freetown. Information from callers was passed to the District Health Management Teams (DHMTs) for appropriate follow-up. DHMTs investigated live alerts, and persons meeting the case definition for Ebola were tested for Ebola virus in an isolation facility. ${ }^{11}$ Persons with a positive test result were transferred to an Ebola treatment unit and persons with a negative test result were either discharged or sent to a non-Ebola-specific health facility. All deaths, independent of Ebola status, received safe and dignified burials following a standardised protocol ${ }^{11}$ that was introduced to minimise the risk of Ebola transmission during burials.
The 117 Call Centre was initially operated by the Ministry of Health and Sanitation (MoHS) with support from the WHO. In September 2014, the MoHS requested eHealth Africa (eHA), a non-governmental organisation, to take over 117 operations and enhance system capacity. eHA increased the overall capacity of the 117 system in terms of personnel, call volume and digital systems, and introduced an internet-based database that was accessible in the districts in which the alerts originated.

Alert and response structures were enhanced with the creation of the National Ebola Response Centre (NERC) in November 2014 and the subsequent extension to each district with consistently structured district Ebola response centres (DERCs). The 117 system was enabled to inform the surveillance and burial desks in the DERCs about alerts through direct software links that allowed operators in the DERCs to access the 117 call data for their district.

A national social mobilisation and communication strategy was launched in September 2014 to encourage use of the 117 system and promote its value, including radio discussion programme, engagements with community leaders and other channels of interactive educational communication. ${ }^{12-15}$ The first national 'House to House' campaign was implemented in 19-21 September 2014 wherein social mobilisation teams promoted use of the 117 system for reporting sick people and deaths in communities. A similar national household visit campaign that lasted 3 days was repeated in March 2015. Other targeted engagement campaigns promoting use of the 117 system $^{16-19}$ were conducted with influential religious leaders ${ }^{20}$ traditional healers ${ }^{21}{ }^{22}$ and women's groups. ${ }^{23}$ Through the 'Zero Ebola Campaign' and 'Operation Northern Push' social mobilisation efforts, promotion of the 117 system was intensified in the districts of Western Area, Port Loko and Kambia, where Ebola transmission was highest during the first half of $2015 .^{24}$ An example of a targeted campaign in these districts include ambulance exhibitions aimed to reduce community fears associated with the ambulance services. ${ }^{25}$ Another example is the Bush-to-Bush Campaign with traditional healers that monitored secret shrines to ensure compliance with the ban on traditional healing practices..$^{21}$ Although social mobilisation and communication likely increased the knowledge about the 117 call number in the population, ${ }^{26} 27$ we do not know the true effect of the campaigns on the use of the system.

\section{INFRASTRUCTURE, PERSONNEL AND COST}

When initially repurposed for the Ebola response in August 2014, the 117 system employed 20 staff who were able to handle a maximum of 1400 calls per week. The initial system encountered frequent software malfunctions and capacity challenges. In September 2014, eHA started improving capacity and infrastructure of the system. Staff numbers increased to 44 in September 2014, 120 in October 2014 
and to a maximum of 198 between December 2014 and March 2015 when the NERC intensified efforts to control Ebola transmission. Staffing levels were decreased as the epidemic waned and eventually stabilised at 52 call operators for all of 2016.

In response to an exponential increase in Ebola cases in Freetown, an intensified response effort called Operation Western Area Surge began in late December $2014,{ }^{28}$ which consisted of a massive expansion in surveillance, laboratory and treatment capacity. During the surge, the 117 system received $>16000$ calls per week.

In response to concern that calls were not acted on, especially early in the outbreak when capacity constraints in the response led to delays in care delivery, a system of callbacks was started in November 2014 whereby callers to 117 were contacted by a team at the 117 centre to ensure their calls had been attended to. A total of three callback attempts were made before a caller was deemed to be unreachable. The proportion of callers to 117 reached by the callback system in January 2015 was $60 \%$ of live alerts and $70 \%$ of death alerts. These proportions remained stable during subsequent months. Lack of phone network coverage in rural areas and individuals switching phones off when not in use were thought to be the main reasons for not reaching callers. Communication between the call centre and the districts was enhanced by placing Alert Coordinators in DERCs who ensured calls were passed to appropriate desks and that any information that came to the DERC without going through 117 was entered into the 117 system.

Following the end of the Ebola epidemic on 7 November 2015, the MoHS decided that all deceased persons should have a buccal swab taken for Ebola testing prior to burial. Only deaths suspicious for Ebola received a safe and dignified burial. The 117 system became the primary mechanism for reporting deaths to the DERC and to DHMTs after the DERCs were wound down in December 2015. Mandatory testing continued until 30 June 2016 when it was replaced by testing only of deaths that were suspicious for Ebola. However, the MoHS continued to encourage people to report all deaths to 117 through press conferences, radio and other media outlets. Live alerts continued to be reported to DHMTs through 117, but received a case investigation only if the district surveillance officer felt the case was possibly due to Ebola.

During the highest level of operations with 198 staff members, the alert system cost approximately US $\$ 200$ 000 per month. Since January 2016, monthly costs have been approximately US\$47 000 . No social mobilisation has been done subsequent to the initial phase to increase death reporting through 117.

\section{ALERT TRENDS OVER TIME}

Figure 1 shows death-alert and live-alert calls to 117 and weekly Ebola case counts reported in WHO situation reports ${ }^{1}$ from September 2014 to November 2015.
Death alerts were highest in the middle of October 2014 (epi week $41 ; \mathrm{n}=9344$ ) just before the peak in weekly new Ebola cases and decreased over time as weekly case numbers decreased nation-wide. Live alerts were highest in late December 2014 (epi week 51; n=3031) just after the peak in weekly new Ebola cases, and similarly decreased as Ebola incidence declined nationwide. The number of death alerts received was consistently higher than live alerts throughout the course of the epidemic, indicating possible missed opportunities to detect and refer suspected Ebola cases for diagnosis and treatment. Between October and December 2014 (epi weeks 40-52), death alerts originating from Western Area accounted for 83\% (75 237 out of 90 217) of calls when the capital, Freetown (situated in Western Area), experienced its highest levels of weekly Ebola cases (table 1), and substantially decreased following implementation of Operation Western Area Surge. Even though only about one-fifth of the population resides in Western Area,${ }^{29}$ it was not until mid-2016 that the proportion of death alerts originating from Western Area decreased to reflect more closely its proportion of the population (tables 1 and 2).

Deaths continued to be reported through the 117 system after the peak of weekly new Ebola cases (November-December 2014) and during the period of enhanced surveillance (November 2015-June 2016). Reporting declined across all geographic regions after the end of the epidemic in November 2015 and even more so after the end of the mandatory testing policy in June 2016. On the other hand, live alerts dramatically reduced across all geographic regions immediately after the peak of weekly new Ebola cases and further after the end of the epidemic.

During the epidemic period, calls to 117 were also made for reasons other than live and death alerts. In September 2014, calls for health information accounted for $16 \%$ of total calls. Other calls not related to live or death alerts accounted for a further $20 \%$ of calls at that time. These included calls to inform authorities of non-Ebola emergencies, such as perinatal emergencies and motor vehicle accidents, as well as enquiries about lifting of Ebola-related restrictions such as reopening of schools. By mid-October 2014, however, calls for reasons other than live and death alerts had decreased to $<10 \%$ of total calls and remained at this level.

\section{CURRENT SITUATION}

Since August 2016, the 117 system has been capturing basic data for cause and place of death. For cause of death, deaths are categorised as acute illness, chronic illness, unexplained, accident, maternal mortality, or homicide. Place of death data are categorised as residence, health facility or public/community. Notifying 117 of deaths is not required to obtain burial permits from the Office of Births and Deaths (OBD). However, for deaths reported through the 117 system, a unique identification number 


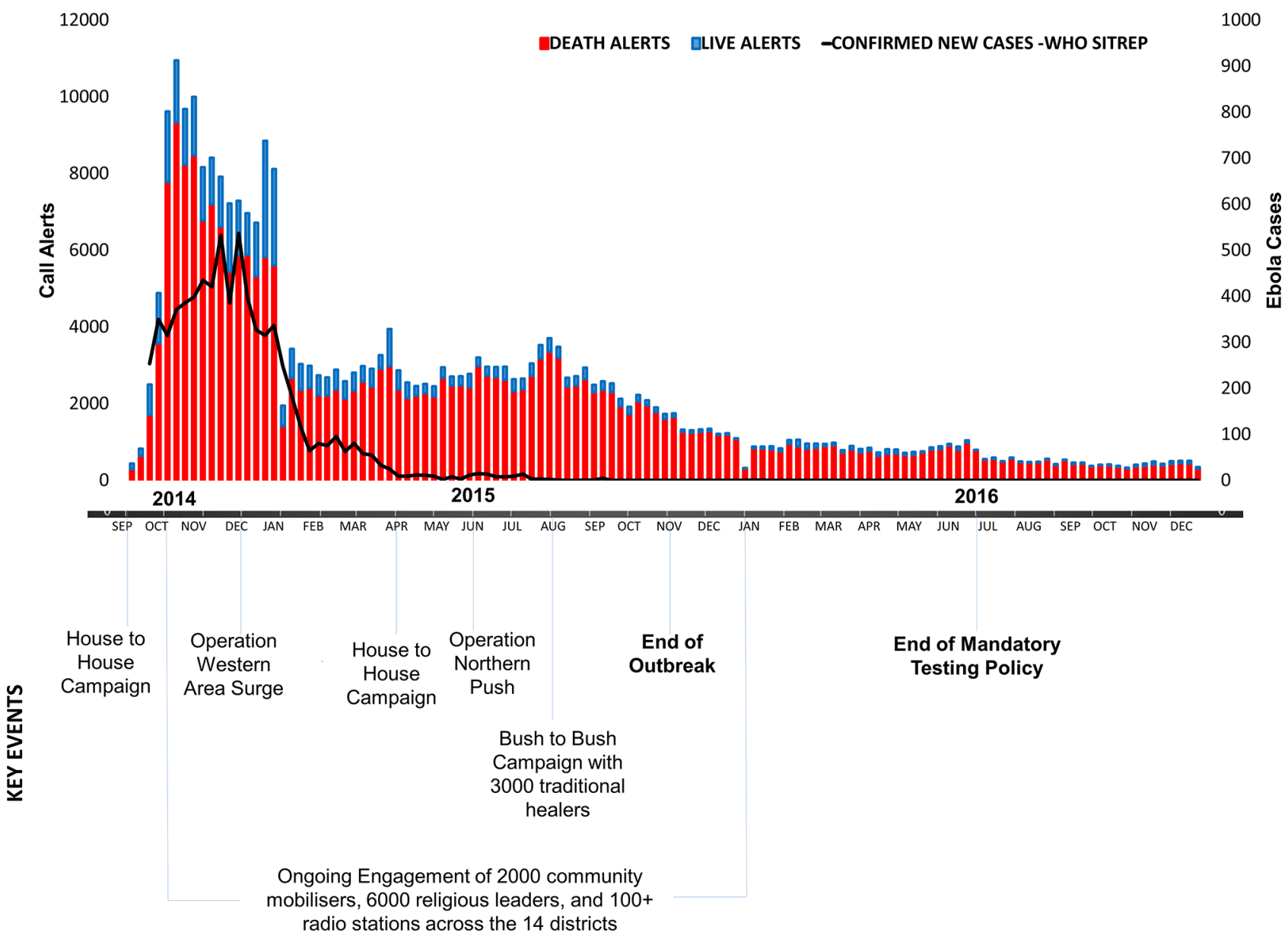

Figure 1 Weekly 117 death- and live-alerts and confirmed new Ebola cases, September 2014 to December 2016, Sierra Leone.

is generated and given to the caller when obtaining burial permits and registering the death with OBD. Outside of death notifications, the 117 system continues to receive calls to inform authorities of emergencies such as a major water main rupture in Freetown in December 2016.

The number of death and live alerts were highest close to the peak of the Ebola epidemic, started to decrease before the peak of the epidemic, and continued to decrease in the post-Ebola period. No further social mobilisation was conducted after the end of the epidemic in November 2015.

In spite of this post-Ebola change in usage, 117 provides a well-known, real-time, national-level and districtlevel mobile-phone health reporting infrastructure in Sierra Leone, and contributes to global health security including enhancing the potential for detection and control of public health threats at their source. We believe that public familiarity with 117 offers an excellent opportunity for the country to remain prepared for another Ebola or similar epidemic. A 2013 survey found that 55\% of households in Sierra Leone (84\% in urban areas) owned a mobile phone. ${ }^{30}$ Phone coverage in the country has likely increased since 2013. The 2016 National Civil Registration Act has established a new authority in Sierra Leone that will be responsible for registration of citizens and residents and recording vital events, including births and deaths. ${ }^{31}$ The act intends to improve death reporting and registration in Sierra Leone. ${ }^{32}$

As of March 2017, Sierra Leone has in place an electronic reporting system for Integrated Disease Surveillance and Response (IDSR) with weekly data from $>95 \%$ of health facilities for all IDSR priority diseases. ${ }^{33}$ Facility-based maternal mortality reporting is part of IDSR reporting ${ }^{34}$ and discussions are ongoing to add under-five deaths. Furthermore, reporting births and deaths is part of the scope of work for community health workers (CHW) in the new CHW policy that is currently being rolled out in Sierra Leone. ${ }^{35}$ CHWs could play a considerable role in death notifications to the 117 system. Notifications could then be transferred through a common data platform to the civil registration and mortality surveillance systems. Maternal deaths are notifiable events and require an investigation of causes and circumstances when they occur in healthcare facilities. ${ }^{36}$ The 117 system could also help improve mortality surveillance for maternal and child deaths occurring outside of health facilities and allow these deaths to be included in cause of death investigations. Technical consultations are currently ongoing to determine minimal infrastructure, staff and funding needs to ensure that such a tool fulfils long-term objectives. 


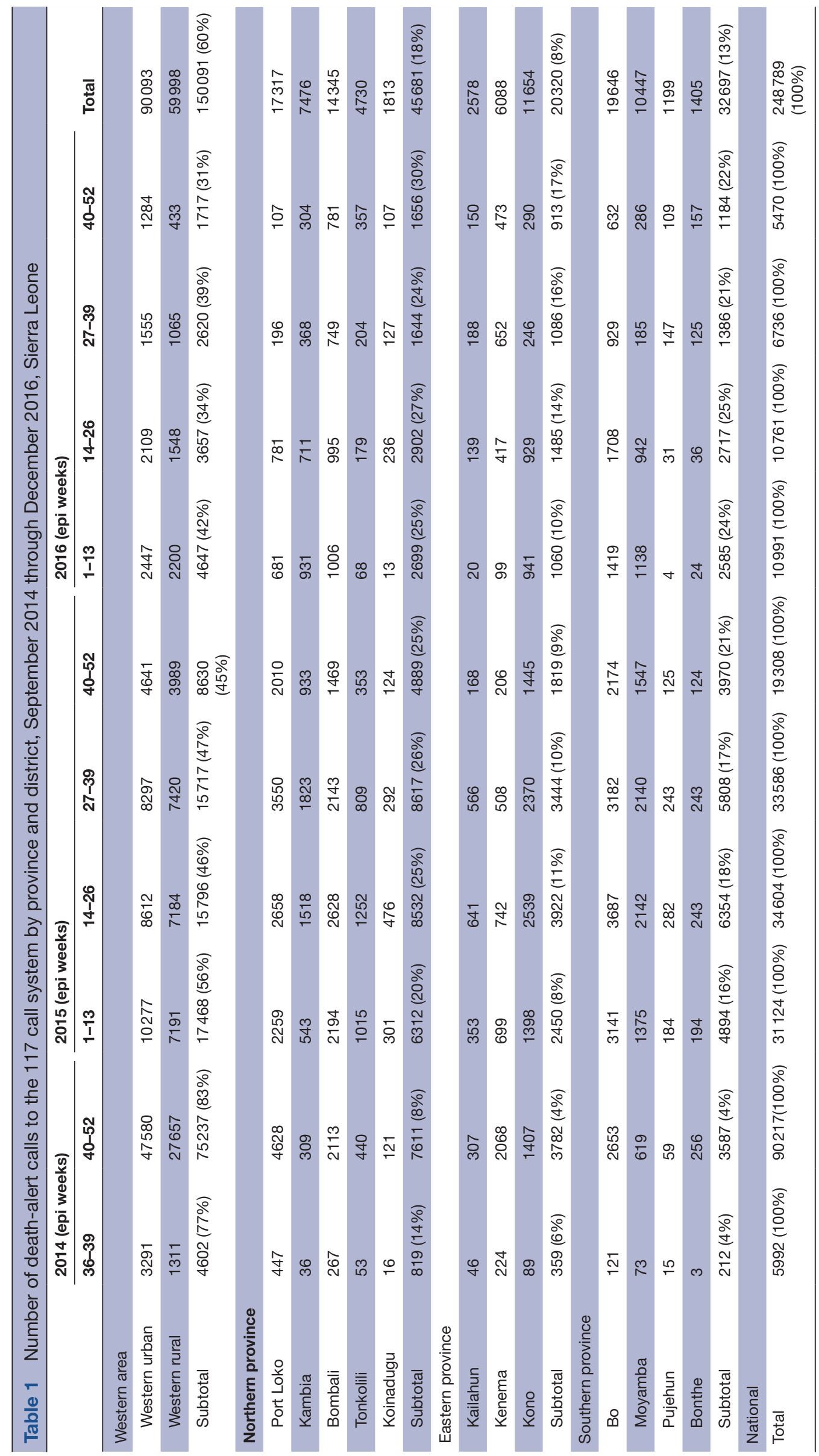




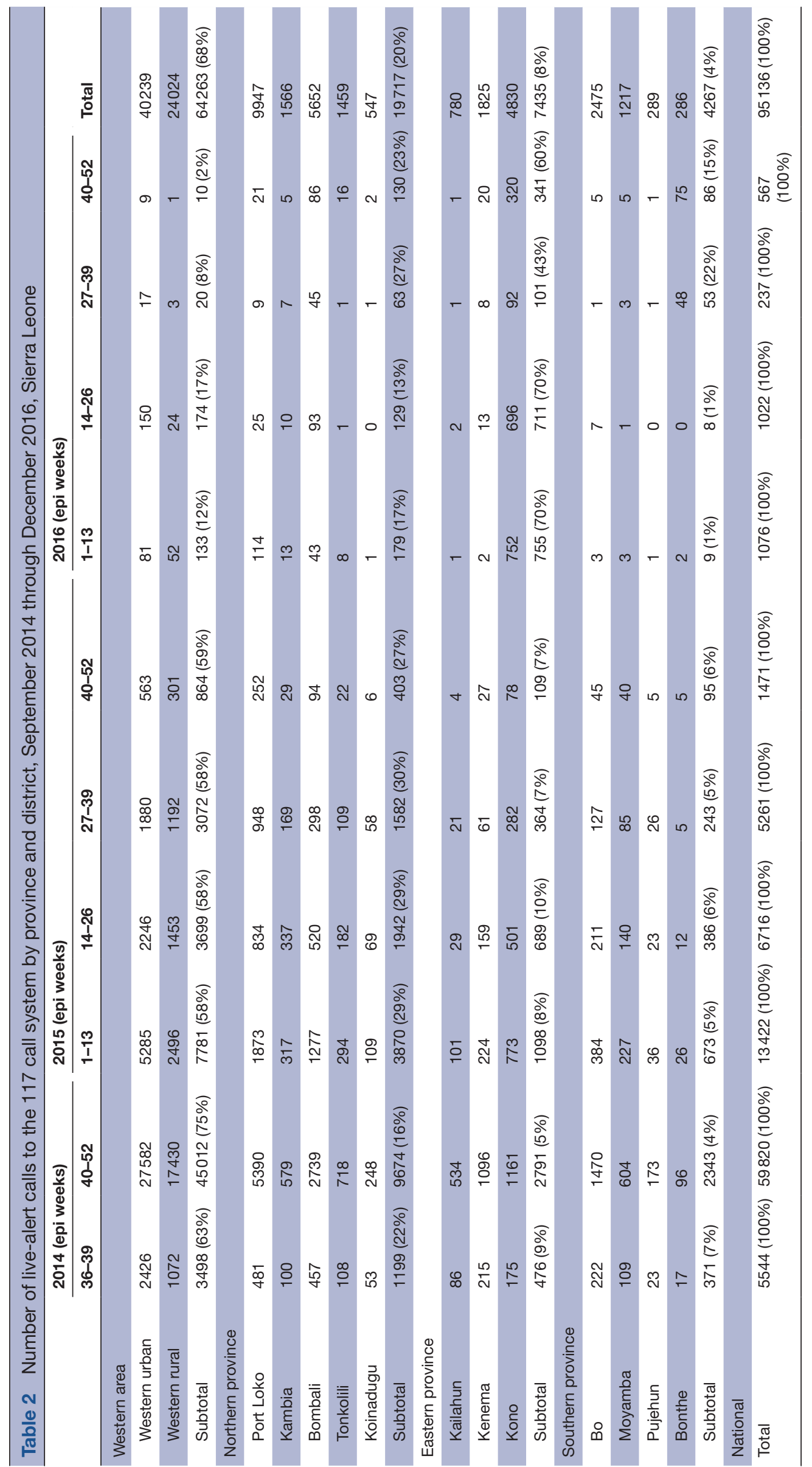


A formative assessment began in March 2017 to identify barriers and motivations for reporting deaths as well as community suggestions for effective death reporting implementation strategies. The assessment will help inform and guide targeted communication and social mobilisation interventions to enhance death reporting in the country and will include questions about previous use of 117 and preference for continuation or discontinuation (Mohamed Jalloh, personal communication). If funding for continued use of the 117 system can be secured, a routine social mobilisation component will be required to increase and maintain usage at an optimal level.

\section{CONCLUSIONS}

The Government of Sierra Leone repurposed the 117 system as an emergency tool for rapid detection of live cases and deaths during and after the 2014-2015 Ebola epidemic. The existing infrastructure and experience with the system offer an opportunity to consider longterm use of the system for routine civil registration and mortality surveillance programme, potentially with a major focus on maternal and child health. Reporting of deaths or clusters of deaths through the 117 system could also contribute to the rapid detection of a public health threats and the system could be used to rapidly control an emerging or re-emerging epidemic. Provided sufficient political will, funding and continuous social mobilisation and community engagement to increase usage, the 117 system could become a model for toll-free, phonebased death reporting tool that could be used in other low-income and middle income countries. Any use of the 117 system as a future death reporting system should be thoroughly monitored and evaluated.

Contributors All coauthors: contributed to the conceptualisation and design of the analysis plan. CA and MFJ led the data analysis with technical support from RK and JTR. All coauthors contributed to the interpretation of the results and preparation of the manuscript.

Funding Several authors are US government employees. This work was conducted as part of authors' normal duties and required no separate funding.

Disclaimer The findings and conclusions in this report are those of the authors and do not necessarily represent the official position of the Centers for Disease Control and Prevention.

\section{Competing interests None declared.}

Ethics approval This analysis was reviewed and approved by Centers for Disease Control and Prevention, Center for Global Health Human Subjects Review Board.

Provenance and peer review Not commissioned; externally peer reviewed.

Open Access This is an Open Access article distributed in accordance with the Creative Commons Attribution Non Commercial (CC BY-NC 4.0) license, which permits others to distribute, remix, adapt, build upon this work non-commercially, and license their derivative works on different terms, provided the original work is properly cited and the use is non-commercial. See: http://creativecommons.org/ licenses/by-nc/4.0/

(c) Article author(s) (or their employer(s) unless otherwise stated in the text of the article) 2017. All rights reserved. No commercial use is permitted unless otherwise expressly granted.

\section{REFERENCES}

1. WHO. Ebola situation reports. http://apps.who.int/iris/bitstream/ 10665/204714/1/ebolasitrep_30mar2016_eng.pdf

2. WHO. Factors that contributed to undetected spread of the Ebola virus and impeded rapid containment. http://www.who.int/csr/ disease/ebola/one-year-report/factors/en

3. Agua-Agum J, Ariyarajah A, Aylward B, et al. Exposure patterns driving Ebola transmission in West Africa: A retrospective observational study. PLoS Med 2016;13:e10002170.

4. Lawrence P, Danet N, Reynard O, et al. Human transmission of Ebola virus. Curr Opin Virol 2017;22:51-8.

5. Elston JWT, Moosa AJ, Moses F, et al. Impact of the Ebola outbreak on health systems and population health in Sierra Leone. $J$ Public Health 2015:fdv158. fdv158.

6. Miller LA, Stanger E, Senesi RG, et al. Use of a nationwide call center for Ebola response and monitoring during a 3-day houseto-house campaign - Sierra Leone, September 2014. MMWR Morb Mortal Wkly Rep 2015;64:28-9.

7. Heymann DL, Chen L, Takemi K, et al. Global health security: the wider lessons from the west African Ebola virus disease epidemic. Lancet 2015;385:1884-901.

8. Frieden TR, Damon IK. Ebola in West Africa-CDC's role in epidemic detection, control, and prevention. Emerg Infect Dis 2015;21:1897-905.

9. Alpren C, Sloan M, Boegler KA, et al. Notes from the field: Ebola Virus Disease Cluster - Northern Sierra Leone, January 2016. MMWR Morb Mortal Wkly Rep 2016;65:681-2.

10. Government of Sierra Leone. Emergency management program standard operating procedure for safe burials. http://nerc.sl/?q= sierra-leone-emergency-management-program-standard-operatingprocedure-safe-dignified-medical

11. Government of Sierra Leone. Bylaws for the prevention of Ebola and other diseases. https://www.humanitarianresponse.info/en/ operations/west-and-central-africa/document/laws-preventionebola-other-diseases-sierra-leone

12. Government of Sierra Leone. National communication strategy for Ebola response in Sierra Leone. http://ebolacommunicationnetwork. org/wp-content/uploads/2014/10/National-Ebola-CommunicationStrategy_FINAL.pdf

13. Government of Sierra Leone. Standard operating procedures for Ebola social mobilization and community engagement. http://nerc. $\mathrm{sl} /$ ?q=sierra-leone-standard-operating-procedures-sops-ebolasocial-mobilisation-and-community-engagement

14. Government of Sierra Leone. Consolidated message guide for Ebola communication in Sierra Leone. http://nerc.sl/sites/default/files/ docs/Consolidated_Message_Guide\%20for\%20Website_0.pdf

15. Gillespie AM, Obregon R, El Asawi R, et al. Social mobilization and community engagement Central to the Ebola Response in West Africa: lessons for future public health emergencies. Glob Health Sci Pract 2016;4:626-46.

16. Government of Sierra Leone. When to call 117. http://nerc.sl/?q= when-call-117-messaging-v2

17. Government of Sierra Leone. Get early treatment for Ebola. http:// nerc.sl/?q=get-early-treatment-ebola

18. Government of Sierra Leone. Allow for safe burial when someone dies at home. http://nerc.sl/?q=allow-safe-burial-when-someonedies-home-\%E2\%80\%93-sierra-leone

19. Government of Sierra Leone. Ebola big idea - a coordinated national media campaign. http://nerc.sl/?q=ebola-messages-sierra-leone

20. FOCUS 1000. Facilitators guide on training of religious leaders on Ebola prevention and control. http://focus $1000.0 \mathrm{rg} /$ index. $\mathrm{php} /$ downloads-resources/summary/11-social-mobilisation/ 26-facilitators-guide-on-training-of-religious-leaders-on-ebolaprevention-and-control

21. FOCUS 1000. Traditional healers on contact tracing guide in the North. http://www.focus1000.org/index.php/blog-1/150-traditionalhealers-on-contact-tracing-drive-in-the-north

22. United Nations. Global Ebola Response Coalition - issues discussed and next steps, 2015. https://ebolaresponse.un.org/sites/default/ files/3july2015.pdf

23. United Nations. Sierra Leonean female journalists add momentum in final push towards zero Ebola. https://ebolaresponse.un.org/sierraleonean-female-journalists-add-momentum-final-push-towardszero-ebola

24. Government of Sierra. Leone. Zero-Ebola: getting to a resilient zero. http://nerc.sl/sites/default/files/docs/20150321\%20Zero\%20Ebola\% 20National\%20Plan.pdf

25. US Centers for Disease Control and Prevention. Destigmatizing ambulances during the Ebola response. http://www.cdc.gov/ globalhealth/countries/sierra-leone/stories/ebola-stories/ destigmatizing_ambulances.htm 
26. FOCUS 1000, UNICEF, and Catholic Relief Services. Public knowledge, attitudes, and practices relating to Ebola Virus Disease prevention and treatment in Sierra Leone, preliminary report. Freetown: FOCUS 1000, 2014

27. FOCUS 1000, US Center for Disease Control and Prevention, and UNICEF. Follow-up study on Public knowledge, attitudes, and practices relating to Ebola Virus Disease prevention and treatment in Sierra Leone, preliminary report. Freetown: FOCUS 1000, 2014.

28. United Nations. Sierra Leone to launch surge response to tackle Ebola transmission. http://ebolaresponse.un.org/un-sierra-leonelaunch-surge-response-tackle-ebola-transmission

29. Statistics Sierra Leone. population and housing census, 2015 https://www.statistics.sl/wp-content/uploads/2016/06/2015-CensusProvisional-Result.pdf

30. Statistics Sierra Leone. 2013 Sierra Leone demographic and health survey. https://dhsprogram.com/pubs/pdf/PR42/PR42.pdf
31. Government of Sierra Leone. National civil registration act 2016 Supplement to Sierra Leone Gazette Vol. CXLVII. 21., 2016.

32. Yonas A, Boeteng I, Calderon C, et al. Interagency technical consultation on improving mortality reporting in Sierra Leone: meeting report. Pan African Med Journal 2017;26:27.

33. US Centers for Disease Control and Prevention. Sierra Leone (IDSR) (GHSA) in action. https://www.cdc.gov/globalhealth/security/stories/ sierra-leone-idsr-ghsa-in-action-story.html

34. WHO. Sierra Leone weekly epidemiological reports. http://www.afro. who.int/en/sierra-leone/data.html

35. Government of Sierra Leone. National community health worker policy 2016-2020. https://www.medbox.org/sierra-leone-nationalcommunity-health-worker-policy-2016-2020/download.pdf

36. Government of Sierra Leone, UNFPA, World Bank, and World Health Organization. Maternal Death surveillance and response: national technical guidelines. 